\title{
NOTE SUR LA TRUITE DE MER
}

\author{
Par M. L. KREITMANN \\ Conservateur des Eilux et Fọrèts.
}

- La Truite (Salmo trulta I.) présente des aspects divers, suivant son haljitat. Ici, elle se confine dans les ruisseaux; sa taille reste alors réduite, sa livrée répète la teinte des fonds sur lesquels elle vit, passant du gris presque noir jusqu'au jaunâtre, parfois mème tachée comme du marbre : mais, toujjours, elle s'avive de points rouge vif auréolés d'orange. ILà, la Truite s'ébat dans les vastes espaces des lacs ou des grands fleuves; les longs parcours influent sur sa taille ; son poids peut dépasser plusieurs kilogrammes ; la couleur de sa robe vire du gris-bleu ou du-gris verdâtre sur le dos à l'argenté sur les flancs et au blanc mat sur le ventre; des taches noires en étoile marquent toujours la moitié supériẹre du corps, et même les opercules et la nageoire dorsale.

- Ces deux formes de la Truite-sont sédentaires; leurs seuls déplacements sont ceux qui, à la fraye, poussent les géniteurs vers l'amont, à la recherche de plaques de fin gravier, dans le lit de leur.rivière d'origine, ou dans des affluents moins : tumultueux.

Mais, côte à côle avec elles, dans le même bassinn, d’autres Truiles obéissent à un instinct de lointaines migrations et; abandonnant de bonne heure leurs zones d'alevinage, elles descendent, le cours des fleuves, franchissent les estuaịrcs et pénètrent dans les eaux salées. C'est la Truite de mer. Ses mours rappellent singulièrement celles du Saumon, mais son aire de distribution est beaucoup moins étendue ; elle ne fréquente guère que les tributaires de la mer du Nord et de la Baltique; elle se rencontre, encore en-France-dans les fleuves côtiers de la Manche, et elle :sc räréfie au delà du Finistère.

Elle paraît se plaire aux lointains périples.; on a en effet capluré vers les :côtes allemandes des individus qui avaient élé marqués, comme Truitelles, en Ecosse où ceite forme abonde. La Truite de mer interrompt aussi assez fréquemment son séjour en mer pour séjourner par intervalles dans les estuaires. Si l'ambiance salée excite son appétit et provoque son développement, les incursions dans les eaux saumâtres ou mêmes douces peuvent le ralentir et la lecture des écailles de la Truitè de mèr et par suite la détermination de son âge et des circonstances. de sa vie ne sont 
pas facilitées par son humeur vagabonde qui la fait passer trop facilement d'un milieu à l'autre.

Elle se distingue du Saumon par des caractères anatomiques ; elle possède, en particulier, un vomer dont la plaque antérieure est triangulaire ou arrondie et porte à sa base une ligne de 3 ou 4 fortes dents et dont la tige est armée d'une rangée de dents incurvées de part et d'autre, et rarement caduques, tout au moins sur la moitié antérieure. Le vomer du Saumon présente au contraire une plaque antérieure pentagonale, dépourvue de dents à tout âge, el une tige allongée dont les dents. rangées en une ligne, tombent peu à peu presque toutes ou même toutes. Le nombre des appendices pyloriques est sensiblement moins élevé chez la Truite que chez le Saumon.

Si l'on s'en rapporte aux caractères extérieurs d'un examen apparemment plus facile, on s'aperçoit que les différences sont assez faibles. La nageoire caudale de la Truite est découpée d'un trait rectiligne, tandis que celle du Saumon est entaillée et se partage en deux lobes ; mais il arrive que cetle distinction manque de nelleté. La bouche est moins grande chez la Truite et ne dépasse guère le bord postérieur de l'œil ; il s'agit là encore d'une proportion parfois incertaine.

Les livrées soṇt semblables ; Ie dos de la Truite de mer est gris-bleuâtre, mais sa couleur peut tirer sur le brun jusque sur les flàncs, le ventre est blanchâtre. La moilié supérieure du corps est marquée de taches noires en étoile, mais celles-ci peuvent aussi manquer plus ou moins. Le Saumon sc pare d'une teinte foncée gris-bleu tachée de noir; les flancs s'éclaircissent et le veritre vire à l'argenté. Les mâles des deux espèces portent, en parure de noce, des points noirs arrondis, auréolés d'orange; ceux-ci sont généralement plus nombreux chez le Saumọn. La déformation de la mandibule inférieure, si caractéristique chez le Saumon mâle auquel elle a fait donner le nom de bécard, se retrouve, mais beaucoup moins accentuée, chez la Truite de mer.

Cielle-ci, nous l'avons dit, passe sa période juvénile dans les eaux douces ; elle se développe pendant son adolescence en mer, tantôt au large, tantôt près des côtes et même dans les estuaires; puis, quand èlle devient adulte et que les prémices de la maturité sexuelle se manifestent, elle reprend en sens inverse son premier voyage et remonte les fleuves à la recherche des frayères sur lesquelles elle pourra se reproduire.

En règle générale, sesł migrations dans les eaux continentales sont courtes; en Allemagne, dans le Rhin, elle atteint à peine l'embouchure du Main, alors que le Saumon, son compagnon, poursuit sa route jusqu'en Suisse, ou plutôt la poursuivait, car rares deviennent les individus qui peuvent encore franchir les obstacles accumulés par l'homme sur sa route et dont le barrage de Kembs est le dernier édilié. A ce point de vue, la Truite de mer, moins exigeante que le Saumon pour la situation de ses frayères, ne manque pas: de présenter un grand intérèt pour la mise en valeur des fleuves qu'elle fréquente et sa propagation mériterail d'ètre suivie avec attention. 
Pourtant, plus on savance vers l'Est, et plus elle remonte vers la tẹtc des bassins ; elle pénètre déjà haut dans. l'elbe et la Fulda el davantage encore dans l'Oder où sa zone de frayères coïncide avec celle du Saumon. Dans la Vistule, elle se sépare mème du Saumon el c'est là une situation particulière que j'ai eu l'occasion de constater récemment et sur laquelte je désirerais retenir l'attention.

La dernière réunion du Comité des Experts du Saumon et de la Truite de-mer constitué par le Conseil International pour l'Exploration de la Mer a eu lieu en Pologne et j'ai eu l'avantage d'y remplacer M. le Profes: sèur Roule, empêché.

Au cours d'une excursion très intéressante dans une région des plus pittoresques des Carpathes polonais, le Professeur Siedeccki, le réputé zoologue de l'Université jagellonienne de Cracovie, a fait parcourir aux membres du Comité la zone des frayères de la Truite de mer sur la. Dunajec. Ceite rivière est uṇ affluent de la vislule dans laquelle elle se jette à quelqué soixante kilomètres en aval et à l'Est de Cracovie: De longueur semblable à leur confluent, toutes deux prennent leur source dans les Carpathes et ne paraissent guère différer. l'une de l'autre. Ciependant, le Saumon évite la Dunajec ét se maintient dans la Vistule qu'il ne remonte d'ailleurs guère plus haut ; la Truite de mer, manifestant une préférence inverse, bilurque toujours dans la Dunajec et continue sá course jusque vers Zakopane, à près de r.ooo mètres d'altitude. Le Professeur Spiczakow, le savant Directeur de l'Institut d'hydrobiologie de Cracovie; a vérifié très exactement les caractères des poissons qui fréquentent ces deux rivières et a pu meltre en évidence, sans conteste, le curieux partage des espèces qui se fait au confluent de la Vistule el de la Dunajec, sans parvenir jusqu'à présent à en découvrir les causes. En plus, on constate ici que, contrairement à ce qui se passe dans les bassins plus occidentaux, la Truite de mer dépasse le Saumon dans sa course vers les sources; elle parcourt plus de r.200 kilomètres depuis l'embouchure de la Vistule jusqu'à ses frayères et il lui faut un an pour accomplir ce long trajet. Aussi est-ce fatiguée par le jeụne et par l'élaḅoration de ses produits-sexuels, qu'elle parvient au terme de son voyage. Les individus sont de belle taille; nous en avons vu de 7 kilogrammes; et c'est là un poids normal.

Dans le désir de conserver et mème de multiplier celte splendide forme de la Truite, les limnologues de Pologne en poursuivent la propagation artificielle ; il est procédé dans ce but à des pêches" exceptionnelles qui ne sont pas sans exiger des qualités spéciales d'endurance el d'habileté de la part du personnel qui en est chargé. Les deux pièges que nous avons visités, à 50 kiloniètres de distance l'un de l'autre, étaient placés dans les endroits où les caux de la rivière sont largement étalécs' en une nappe mince et régulière. Les gardes disposent, d'une rive à l'autre, un barrage de claies, dont les brins sont assez rapprochés pour s'opposer au passage du poisson et assez écartés pour laisser l'eau s'écoulèr. Ces claies sont liées à des pieux fichés dans le lit de. la rivière et elles sont maintenues 
incilinéés pai des fourches ; celles-ci sont enlevées el les claies rabattues, si les eaux deviennent hautes; il n'est cependant pas rare què l'ouvrage soit emporté et doive être refait 2 ou 3 fois au cours de la saison. La Truite qui vient buter contre le barrage est dirigée vers l'ouvcrturc d'une pêcherie dite " jardinet." où les gardes la capturent à la trouble. Ils pratiquent ces diverses opérations en marchant dans l'eau et la froidure d'un automne précoce ne paraît pas les émouvoir ni les empĉcher d'exercer leur'surveillance de jour et de nuil, sur la rive, dans une baraque ouverte à tous les vents.

Les poissons caplurés sont transportés vivants dans les établissements de pisciculture où ils sont entreposés dans des bassins jusqu'à leur maturité sexuelle. Nous avons visilé ceux de Nowy Sacz et de Nowy Targ où la plus aimable hospitalité nous a été offerte par le Fishing Club de Pologne et son Président, le Professeur Jujjan Nowak. Chacun de ces établissements contient un certain nombre de bacs d'incubation et d'alevinage des types habituels et peut traiter de I à 2 millions d'œufs; mais un meuble qui frappe la vue dès l'entrée et qui est tout à fait inattendu, est un énorme poêle de fä̈ence, nécessaire dậs ces régions où les rigueurs de l'hiver provoqueraient un gel néfaste si elles n'étaient tempérées. La récolte qui assure l'approvisionnement en oufs est assez variable ; car la capture des géniteurs passe de 35 à 300 individus suivant les années ; la hauteur des eaux, l'aménagement des barrages et l'habileté des gardes interviennent pour beaucoup dans la rúussite de la campagne de pêche et, de toute façon, un grand nombre de sujets échappe aux engins et va s'installer sur les frayères naturelles.

Au cours de son long voyage de la Baltique aux Carpathes, la Truite de mer est exposée aux risques de la pêche ; mais c'est surlout à l'embouchure de.la Vistule que les filets sont les plus meurtriers pour elle. La propagation artificielle dont nous venons de parler s'efforce de remédier à ces abus ; son utilité est incontestable quand il s'agit de repeupler des eaux dont la Truite est absente ou qu'elle a abandonnées ; le réempoissonnement très réussi de la Raba en est une preuve évidente. Cependant, les frais de telles opérations restent très élevés, même dans un pays où la main-d'œuvre est très peu coûteuse, et l'on est en droit de se demander si une réglementation restrictive dans les eaux de l'Etat libre de Dantzign ne pourrait aussi bien contribuer au maintien et-même au développement de la Truite de mer dans toute l'étendue du bassin de la Vistule.

Quoi qu'il en soit, en présence de l'intérêt et de l'importance que présente la pêche de la Truite de mer en Pologne, il nous a paru opportun d'appeler l'attention sur ce poisson. Sa propagation pourrajt être poursuivie utilement dans les fleuves côtiers de la Manche qu'il fréquente, pour la meilleure mise en valeur de leurs eaux et pour la plus grande satisfaction des pêcheurs. 